Revista Latinoamericana de la Papa 19 (1): 18-28

ISSN: 1853-4961

http://www.papaslatinas.org/revista.html

\title{
Eficiencia de ametoctradin en el control del tizón tardío de la papa [Phytophthora infestans (Mont.) De Bary] en condiciones de Argentina
}

\author{
J. D. Mantecón ${ }^{1}$
}

Recibido: $21 / 10 / 2014$

Aceptado: $11 / 04 / 2015$

Disponible en línea: Junio 2015

\section{Resumen}

Durante las campañas agrícolas 2008/09, 2009/10 y 2010/11 se realizaron tres ensayos de control del tizón tardío de la papa (Phytophthora infestans) mediante un programa de pulverizaciones de fungicidas foliares utilizando semilla de alta sanidad del cultivar Kennebec, susceptible a la enfermedad. El diseño utilizado fue en bloques completos aleatorizados con cuatro repeticiones. La incidencia de la enfermedad se evaluó en base a síntomas en el follaje y rendimiento de papa recibo (mayor a $60 \mathrm{~g}$ ), "semilla" (menor a $60 \mathrm{~g}$ ) y total de los dos surcos centrales de cada parcela. Todos los tratamientos químicos lograron reducir los síntomas patológicos en el campo, pero la mayor eficiencia se observó con BAS 65100F 525SC (ametoctradin + dimetomorph), Acrobat MZ 90/600 y BAS 65200F 56XL (ametoctradin + metiran) en la menor dosis, pulverizados semanalmente. Se detectaron diferencias entre intervalo de aplicación para BAS 65100F 525SC en una misma dosis o dosis mayor. Todos los tratamientos químicos superaron al testigo sin pulverizar en rendimiento, pero los mayores valores en tubérculos comerciales se obtuvieron con BAS 65100F 525SC pulverizado cada 7 días. Se detectaron diferencias significativas entre tratamientos en el rendimiento tubérculos "semilla". Los aumentos de rendimiento de tubérculos comerciales alcanzados con BAS 65100F 525SC durante 2008/09, 2009/10 y 2010/11 llegan a valores del $93 \%, 80 \%$ y $93.5 \%$, respectivamente.

Palabras clave adicionales: Fungicidas, rendimiento, dosis, tubérculos, síntomas.

\section{Ametoctradin efficiency for potato late blight [Phytophthora infestans (Mont.) De Bary] control in Argentinian conditions}

\section{Summary}

During 2008-09 to 2010/11 seasons three trials late blight control (Phytophthora infestans) were performed using a program of foliar fungicide sprays with highly healthy seed of the Kennebec cultivar which is susceptible to the disease. The design was completely randomized blocks with four repetitions. The incidence of the disease was assessed based on symptoms in the foliage and marketable (greater than $60 \mathrm{~g}$ ) and seed potato yield of the two central rows of each plot. All chemical treatments were able to reduce disease symptoms in the field, but the highest efficiency was observed with BAS 65100F 525SC (ametoctradin +dimethomorph), Acrobat MZ BAS 90/600 and 65200F 56XL (ametoctradin + metiran) at the lowest dose, all of them sprayed weekly. Differences between application interval for BAS 65100F 525SC in the same dose or higher doses were detected. All chemical treatments exceeded the control unsprayed yield, but higher values in commercial tubers were obtained with BAS $65100 \mathrm{~F}$ 525SC spray every 7 days. Significant differences between treatments in seeds tuber yield

Autor para correspondencia. Correo electrónico: mantecon.jorge@inta.gob.ar Integrada FCA-INTA, cc 276-Balcarce, Argentina. 
were detected. Increases in yield of marketable tubers reached with BAS 65100F 525SC during the trials reach values of $93 \%, 80 \%$ and $93.5 \%$.

Additional keywords: Fungicides, performance, dose, tubers, symptoms.

\section{Introducción.}

El tizón tardío de la papa causado por Phytophthora infestans (Mont.) De Bary es la enfermedad fúngica más grave en las distintas zonas productoras del mundo (Cox y Large, 1960). Está presente en todas las áreas paperas y es considerada como la más importante porque si los campos no están protegidos adecuadamente con aplicaciones planificadas de fungicidas y las condiciones ambientales son óptimas para el desarrollo del patógeno (temperatura de 12 a $15^{\circ} \mathrm{C}$ y humedad relativa de 95 a $100 \%$ ), los cultivos de papa pueden ser destruidos en 10 a 15 días.

El origen de la enfermedad en EUA y en Europa se atribuye a papas silvestres enfermas introducidas desde México (Fry et al., 1992; Fry et al., 1993; Niederhauser, 1991; French et al., 1994). La enfermedad fue reportada en Europa, primero en Noruega en 1841, luego, en EUA en 1842 (en campos de papa cercanos a los puertos de Filadelfia y Nueva York) y en ese mismo año, también se reportó en seis países de Europa (Bélgica, Dinamarca, Inglaterra, Alemania, Irlanda y Escocia). Posteriormente, entre 1845 y 1847, todos los campos de papa cultivados especialmente en Irlanda y en otros países fueron destruidos por esta devastadora enfermedad, ocasionando en Europa

la hambruna más grande de todos los tiempos, donde murieron aproximadamente un millón de personas y otro millón emigró a EUA. Según Niederhauser (1991), en Sudamérica, la enfermedad fue reportada en Argentina por Rieder en 1887 y en Brasil por Potel en 1900, atribuyéndose la presencia en estos dos países a la introducción de papas procedente de Europa en los años de 1880.
No obstante ello, los reportes de epifitias severas comienzan a partir de 1940.

La papa (Solanum tuberosum L.), el pepino (Solanum muricatum Ait.) y el tomate (Lycopersicon esculentum Mill.) son las plantas hospedantes más importantes de Phytophthora infestans. Ocasionalmente afecta a la berenjena (Solanum melongena) y a muchas otras especies de la familia Solanaceae (Abad et al., 1995).

El nombre de Phytophthora deriva de dos voces griegas: Phyto $=$ planta $\mathrm{y}$ phthora $=$ destructor, por lo tanto Phytophthora, significa destructor de plantas.

La presencia de 13 razas de $P$. infestans, hasta ahora identificadas, la diseminación (alrededor del mundo) de los aislamientos del grupo A2 iniciada a partir de 1980, la unión de los dos grupos de apareamiento A1 y A2 para formar estructuras sexuales, han dado lugar a la aparición de aislamientos más agresivos tanto del grupo A1 como del A2. Además, los estudios llevados a cabo por Andrivon (1995), sugieren que la mutación y la migración del patógeno son los factores que más influyen en la evolución de las razas.

El patógeno sobrevive de una campaña a otra, por medio del micelio presente en los tubérculos infectados que han sido eliminados y/o abandonados en el campo o que no fueron cosechados por el agricultor porque el cultivo fue destruido por la enfermedad.

La infección de las plantas de papa en el campo se realiza por medio de los esporangios, los que se encuentran en el medio ambiente y son transportados por la neblina, las nubes y el viento a distancias considerables.

El patógeno causa daños de distinta intensidad en estrecha relación con las condiciones ambientales que se presentan 
durante el desarrollo del cultivo (Carmona, et. al., 2003; Huarte et. al., 1993). Cuando los esporangios llegan a un cultivo de papa, se posan y establecen en las hojas y/o en el lugar de inserción de la hoja con el tallo. Generalmente, la infección en las hojas se produce en el ápice y en los bordes de los foliolos, donde casi siempre existe una película de agua.

La producción de los esporangios es más rápida a $100 \%$ de humedad relativa y a $21^{\circ} \mathrm{C}$ de temperatura. La penetración del patógeno ocurre entre 10 a $29^{\circ} \mathrm{C}$ y el desarrollo de la enfermedad es más rápido a $21^{\circ} \mathrm{C}$. Cuando existen las condiciones ambientales óptimas de temperatura (12 a $15^{\circ} \mathrm{C}$ ) y humedad relativa (95 a $\left.100 \%\right)$, se producen hasta 20 zoosporas (estructuras propagativas) en el interior de los esporangios. Las zoosporas tienen 2 flagelos con los cuales se movilizan nadando en la película de agua existente en la superficie de las hojas o de los tallos. Después de un tiempo, pierden los flagelos, se enquistan, producen un tubo germinativo, forman apresorios $y$ finalmente, los tubos germinativos ingresan a la hoja a través de los estomas o directamente por la cutícula. Una vez que el patógeno se encuentra en el interior de los tejidos forma los haustorios que ingresan dentro de las células para extraer los nutrientes que se encuentran en el protoplasma. Como consecuencia, las células mueren, los tejidos se necrosan y las hojas infectadas muestran manchas negras o marrones.

La infección de los tubérculos se realiza por medio de los esporangios que se encuentran en el envés de las hojas enfermas. Estos esporangios son desprendidos o lavados por las gotas de lluvia y son transportados de las hojas hacia el suelo, donde infectan a los tubérculos que se encuentran cerca de la superficie, especialmente aquellos de las plantas que no han sido adecuadamente aporcadas. Otros factores como la humedad del suelo, la cantidad de esporangios viables que caen, la temperatura del suelo y la susceptibilidad de la variedad, favorecen la infección de los tubérculos en el campo.

El mayor efecto que causa esta enfermedad es la disminución de la cantidad y calidad de los tubérculos comerciales (Mantecón, 2007 y 2009). Los tubérculos cosechados que se hallan infectados resultan poco apropiados para almacenarlos en cámaras, ya que la enfermedad permanece latente a bajas temperaturas y se reactiva cuando los tubérculos son retirados del frío. Cuando éstos tubérculos sean plantados constituirán el inoculo primario para infectar un nuevo cultivo.

Los síntomas en el follaje se caracterizan por lesiones de color castaño oscuro y aspecto húmedo, que en pocos días se vuelven más claras cuando están secas (Hooker, 1980).

No existe una única herramienta para controlar el tizón tardío en los cultivos de papa. Se necesita un programa de manejo integrado de varias técnicas que reduzcan el inoculo. Las prácticas culturales son importantes para disminuir los daños ocasionados por la enfermedad.

La utilización de cultivares de papa con resistencia cuantitativa u horizontal, el uso de "semilla" sana, eliminar todas las fuentes de infección como tubérculos enfermos que se encuentran en los almacenes y plantas voluntarias en los campos además de realizar aporques altos con la finalidad de cubrir adecuadamente con tierra los tubérculos que se encuentran en el suelo, contribuyen a oprimir los efectos de la enfermedad en el cultivo.

Es importante también, evitar la cosecha en días lluviosos, porque los esporangios que se encuentran en el aire, al ponerse en contacto con los tubérculos los infectan y posteriormente la enfermedad se desarrolla en el almacenaje.

Estas estrategias de control tienen como objetivo impedir el desarrollo inicial de la 
enfermedad en los cultivos (Mantecón, 2006, 2006a, 2007, 2007a, 2009, 2009a y 2009b).

El uso de fungicidas para el control de la enfermedad es la estrategia más difundida y aceptada por los productores de Argentina. La dosis y la frecuencia de aplicación dependen del fungicida y su formulación, de las condiciones climáticas $\mathrm{y}$ del nivel de resistencia del cultivar utilizado.

El objetivo del presente trabajo fue evaluar la eficiencia de un nuevo fungicida, ametoctradin formulado en mezcla con dimetomorph (BAS 65100F 525SC) y con metiran (BAS 65200F 56XL) en el control del tizón tardío de la papa (Phytophthora infestans) en condiciones agroecológicas del sudeste bonaerense, Argentina.

\section{Materiales y Métodos}

Durante las campañas agrícolas 2008/09, $2009 / 10$ y $2010 / 11$ se realizaron tres ensayos de control del tizón tardío de la papa mediante un programa de pulverizaciones de fungicidas foliares. Todos los ensayos fueron realizados utilizando semilla de alta sanidad del cultivar Kennebec, susceptible a la enfermedad, fertilizados y regado mediante sistema de aspersión. Fueron plantados con una máquina convencional el 23-11-08, 25-11-09 y 2-12-2010, respectivamente; ejecutando la cosecha manual de la parcelas el 24-04-09, 12-04-10 y 18-042011, respectivamente.

El diseño utilizado fue en bloques completos aleatorizados con cuatro repeticiones; las parcelas fueron de 4 surcos de cuatro metros de largo distanciados a 0.8 metros entre sí. La distancia entre plantas a lo largo del surco fue 0.2 metros. Cada parcela se distanciaba de su vecina por 2 metros en el sentido de los surcos y los espacios entre parcelas no estaban ocupados por plantas de papa.

En todos los ensayos se plantaron cuatro surcos de papa separando cada bloque que nunca fueron pulverizados con fungicidas y donde se realizó la inoculación artificial mediante la incorporación al azar de tubérculos previamente infectados con el patógeno; con el fin de asegurar la incorporación homogénea del inoculo primario de la enfermedad en los ensayos.

El lugar donde se realizaron los ensayos es un sector del campo experimental donde la rotación papa-trigo-soja-papa es una rutina normal de manejo; por lo cual, el suelo está contaminado naturalmente con el patógeno, ya que un cultivo de papa se desarrolla en el mismo lugar sistemáticamente cada dos campañas agrícolas.

Los fungicidas evaluados y sus correspondientes dosis e intervalos entre aplicaciones se detallan en la tabla 1. Los mismos fueron pulverizados sobre las parcelas, utilizando una mochila de presión constante con fuente de $\mathrm{CO} 2$, equipada con una barra de cuatro pastillas cerámicas tipo cono hueco distanciadas a $0.40 \mathrm{~m}$ entre sí, las que, a una presión de $40 \mathrm{lb} / \mathrm{pulg} 2$, arrojaban un volumen de 240 1/ha. 
Tabla 1. Fungicidas, dosis, número e intervalo entre aplicaciones de los tratamientos evaluados en las campañas 2008/09, 2009/10 y 2010/11.

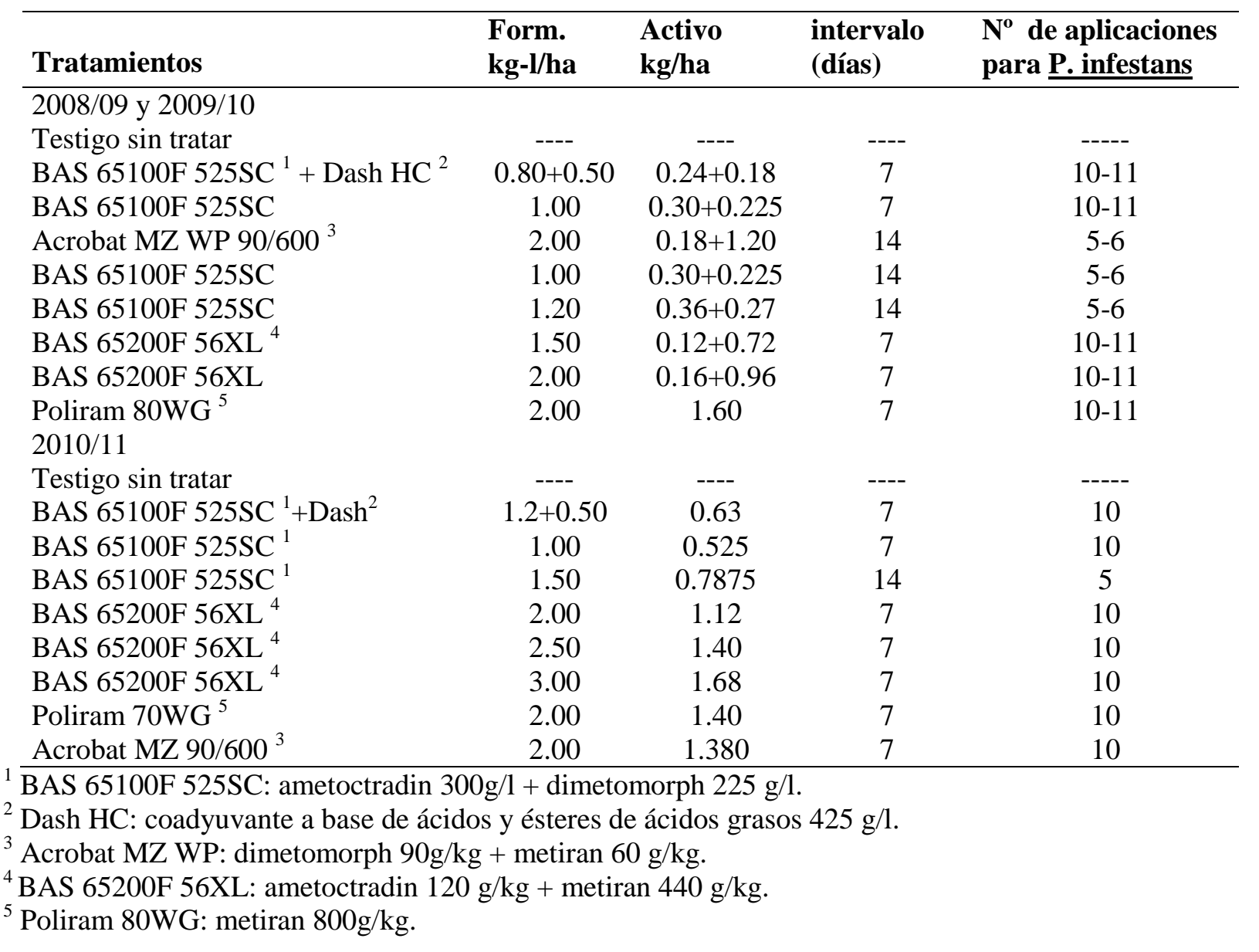

En la tabla 2 se detalla la frecuencia de pulverización de los fungicidas y las lluvias y/o riegos registrados durante ciclo de cultivo en los tres años de evaluación.

La incidencia de la enfermedad se evaluó en base a síntomas en el follaje y rendimiento de papa recibo (mayor a $60 \mathrm{~g}$ ), "semilla" (menor a $60 \mathrm{~g}$ ) y total, de los dos surcos centrales de cada parcela. Para la evaluación de los síntomas en el follaje se utilizó una escala que asigna el valor 0 a la falta total de síntomas y 5 a la máxima infección. En todos los casos no se consideraron los dos surcos externos de cada parcela. 
Tabla 2. Evolución de síntomas de tizón tardío (P. infestans) en el follaje* 2008/09.

\begin{tabular}{|c|c|c|c|c|c|}
\hline \multirow[b]{2}{*}{ Tratamientos - intervalo } & \multicolumn{5}{|c|}{ fecha de evaluación y días de cultivo* } \\
\hline & $\begin{array}{l}\text { Dosis } \\
\text { Form. } \\
\mathrm{Kg} / \mathrm{ha}\end{array}$ & $\begin{array}{c}2-02-09 \\
(71)\end{array}$ & $\begin{array}{c}10-02-09 \\
(79)\end{array}$ & $\begin{array}{c}\text { 24-02-09 } \\
\text { (93) }\end{array}$ & $\begin{array}{c}10-03-09 \\
(107)\end{array}$ \\
\hline Testigo sin tratar & ---- & $1.0 \mathrm{a}$ & $1.5 \mathrm{a}$ & $3.0 \mathrm{a}$ & $5.0 \mathrm{a}$ \\
\hline BAS 65100F 525SC+Dash 7d & $0.80+0.50$ & $0.0 \mathrm{~b}$ & $0.5 \mathrm{~b}$ & $1.5 \mathrm{~b}$ & $2.0 \mathrm{bcd}$ \\
\hline BAS 65100F 525SC 7d & 1.00 & $0.0 \mathrm{~b}$ & $0.5 \mathrm{~b}$ & $0.5 \mathrm{c}$ & $1.0 \mathrm{~d}$ \\
\hline Acrobat MZ 90/600 14d & 2.00 & $0.0 \mathrm{~b}$ & $0.5 \mathrm{~b}$ & $1.0 \mathrm{bc}$ & $2.0 \mathrm{bcd}$ \\
\hline BAS $65100 F 525 S C$ 14d & 1.00 & $0.0 \mathrm{~b}$ & $0.5 \mathrm{~b}$ & $1.5 \mathrm{~b}$ & $3.5 \mathrm{~b}$ \\
\hline BAS $65100 \mathrm{~F} 525 \mathrm{SC} 14 \mathrm{~d}$ & 1.20 & $0.0 \mathrm{~b}$ & $0.5 \mathrm{~b}$ & $1.0 \mathrm{bc}$ & $2.5 \mathrm{bc}$ \\
\hline BAS 65200F 56XL 7d & 1.50 & $0.0 \mathrm{~b}$ & $0.5 \mathrm{~b}$ & $1.5 \mathrm{~b}$ & $2.0 \mathrm{~cd}$ \\
\hline BAS 65200F 56XL 7d & 2.00 & $0.0 \mathrm{~b}$ & $0.5 \mathrm{~b}$ & $1.0 \mathrm{bc}$ & $3.0 \mathrm{bc}$ \\
\hline Poliram 80WG 7d & 2.00 & $0.0 \mathrm{~b}$ & $0.5 \mathrm{~b}$ & $1.0 \mathrm{bc}$ & $2.5 \mathrm{~b}$ \\
\hline
\end{tabular}

* Escala basada en el \% de infección foliar y/o defoliación.

$0=$ sin síntomas; 5 = máxima infección.

** Los promedios que tienen letras en común no difieren entre sí ( $\mathrm{P}=0.05)$.

Se realizó un análisis de la varianza de los datos y los promedios fueron comparados utilizando el test MRT de Duncan con un nivel de significancia del cinco por ciento.

\section{Resultados y Discusión.}

Durante los tres años de evaluación las condiciones ambientales fueron muy favorables para el desarrollo de la enfermedad.

Debido a la implementación de inoculaciones artificiales en el momento de la plantación y el "manejo" del cultivo; en la campaña 2008/09, la enfermedad comienza a desarrollarse en el testigo sin tratar desde los 70 días de cultivo (tabla 2, $1^{\text {a }}$ evaluación), siendo constante su

desarrollo hasta la $3^{a}$ evaluación (90 días de cultivo), alcanzando los mayores niveles de infección una vez superados los 100 días de cultivo.

En la campaña 2009/10 se registró un proceso similar con condiciones climáticas muy favorables (tabla 3), especialmente durante la segunda mitad de enero (65 días de cultivo) donde las lluvias se hicieron más frecuentes provocando el desarrollo de la enfermedad en el campo; mientras que en la campaña 2010/11 la enfermedad se inicia en el testigo sin tratar desde los 60 días de cultivo ( $11^{\mathrm{a} e v a l u a c i o ́ n ~ t a b l a ~} 3$ ), siendo persistente su progreso hasta adquirir los mayores niveles de infección muy temprano en el cultivo, a los 85 días.

Tabla 3. Evolución de síntomas de tizón tardío (P. infestans) en el follaje* 2009/10.

\begin{tabular}{lccccccc}
\hline & \multicolumn{7}{c}{ fecha de evaluación y días de cultivo* } \\
\hline Tratamientos-intervalo & $\begin{array}{l}\text { Form. } \\
\text { Kg/ha }\end{array}$ & $\begin{array}{c}29-1-10 \\
(65)\end{array}$ & $\begin{array}{c}8-2-10 \\
(75)\end{array}$ & $\begin{array}{c}18-2-10 \\
(85)\end{array}$ & $\begin{array}{c}26-2-10 \\
(93)\end{array}$ & $\begin{array}{c}12-3-10 \\
(107)\end{array}$ \\
\hline Testigo sin tratar & ---- & $1.0 \mathrm{a}$ & $1.5 \mathrm{a}$ & $2.5 \mathrm{a}$ & $4.0 \mathrm{a}$ & $5.0 \mathrm{a}$ \\
BAS 65100F 525SC+Dash 7d & $0.80+0.50$ & $0.0 \mathrm{~b}$ & $0.5 \mathrm{~b}$ & $1.5 \mathrm{~b}$ & $3.5 \mathrm{a}$ & $4.5 \mathrm{a}$ \\
BAS 65100F 525SC 7d & 1.00 & $0.0 \mathrm{~b}$ & $0.5 \mathrm{~b}$ & $0.5 \mathrm{c}$ & $1.5 \mathrm{c}$ & 1.5 & $\mathrm{~d}$ \\
Acrobat MZ 90/600 14d & 2.00 & $0.0 \mathrm{~b}$ & $0.5 \mathrm{~b}$ & $1.5 \mathrm{~b}$ & $2.0 \mathrm{bc}$ & $3.0 \mathrm{bc}$ \\
BAS 65100F 525SC 14d & 1.00 & $0.0 \mathrm{~b}$ & $0.5 \mathrm{~b}$ & $1.5 \mathrm{~b}$ & $3.5 \mathrm{a}$ & $3.5 \mathrm{~b}$ \\
BAS 65100F 525SC 14d & 1.20 & $0.0 \mathrm{~b}$ & $0.5 \mathrm{~b}$ & $1.0 \mathrm{bc}$ & $2.5 \mathrm{~b}$ & $3.0 \mathrm{bc}$ \\
BAS 65200F 56XL 7d & 1.50 & $0.0 \mathrm{~b}$ & $0.5 \mathrm{~b}$ & $1.5 \mathrm{~b}$ & $2.0 \mathrm{bc}$ & 2.5 & $\mathrm{c}$ \\
BAS 65200F 56XL 7d & 2.00 & $0.0 \mathrm{~b}$ & $0.5 \mathrm{~b}$ & $1.5 \mathrm{~b}$ & $2.5 \mathrm{~b}$ & $3.0 \mathrm{bc}$ \\
Poliram 80WG 7d & 2.00 & $0.0 \mathrm{~b}$ & $0.5 \mathrm{~b}$ & $1.0 \mathrm{bc}$ & $2.5 \mathrm{~b}$ & $3.5 \mathrm{~b}$ \\
\hline
\end{tabular}

* Escala basada en el \% de infección foliar y/o defoliación.

0 = sin síntomas; 5 = máxima infección.

** Los promedios que tienen letras en común no difieren entre sí $(\mathrm{P}=0.05)$. 
Cuando analizamos la evolución de los síntomas en el follaje en la campaña 2008/09 todos los tratamientos químicos superaron al testigo sin tratar y redujeron los síntomas de la enfermedad en el campo hasta los 79 días de cultivo. Pasados los 100 días de cultivo, el testigo sin tratar alcanzó los mayores valores de severidad y al final del ensayo la mejor eficiencia de control de la enfermedad se observó con los tratamientos de BAS 65100F 525SC, Acrobat MZ 90/600 y BAS 65200F 56XL en la menor dosis; todos ellos pulverizados cada 7 días. Se detectaron claras diferencias entre los intervalos de aplicación para BAS 65100F 525SC en una misma dosis o dosis mayor, por lo cual, parecería que para intervalos de 14 días, BAS 65100F 525SC necesitaría mayor dosis para aumentar la eficiencia de control de síntomas patológicos.

$\mathrm{Al}$ analizar la campaña 2009/10, en la $1^{\mathrm{a}}$ y $2^{a}$ evaluación, los fungicidas evaluados superaron al testigo sin tratar y redujeron los síntomas de la enfermedad en el campo, no registrándose diferencias significativas entre ellos. Al final del ensayo, a excepción de BAS 65100F 525SC en la menor dosis, todos lograron reducir los síntomas patológicos, pero la mayor eficiencia de control se registró con los tratamientos de BAS 65100F 525SC en la mayor dosis pulverizado cada 7 días.

Como en 2008/09, se detectaron diferencias entre los intervalos de aplicación para BAS 65100F 525SC en una misma dosis o dosis mayor. Cuando las condiciones climáticas son altamente conducentes para el tizón tardío, intervalos entre aplicaciones prolongados parecen excesivos para mantener un control eficiente del patógeno en las dosis evaluadas. Adicionalmente, bajo estas condiciones se observa que la eficiencia de control de los fungicidas sistémicos es mayor comparada con los fungicidas no sistémicos.

Durante 2010/11 (tabla 4), todos los tratamientos químicos superaron al testigo sin tratar y redujeron los niveles de infección en la primera evaluación. Nuevamente se detectaron diferencias significativas entre intervalos de aplicaciones para BAS 65100F 525SC. Este resultado, observado durante los tres años de evaluación, muestra claramente que el fungicida fue muy eficaz en intervalos de aplicación de 7 días.

Tabla 4. Evolución de síntomas de tizón tardío (P. infestans) en el follaje* 2010/11.

\begin{tabular}{|c|c|c|c|c|c|c|c|}
\hline \multirow[b]{2}{*}{ Tratamientos-intervalo } & \multicolumn{6}{|c|}{ fecha de evaluación y días de cultivo* } & \multirow[b]{2}{*}{$\begin{array}{c}10-3-11 \\
(98)\end{array}$} \\
\hline & $\begin{array}{l}\text { Form. } \\
\mathrm{Kg} \text { /ha }\end{array}$ & $\begin{array}{c}31-1-11 \\
(60)\end{array}$ & $\begin{array}{c}15-2-11 \\
(75)\end{array}$ & & $\begin{array}{l}2-11 \\
5)\end{array}$ & $\begin{array}{c}1-3-11 \\
(89)\end{array}$ & \\
\hline Testigo sin tratar & --- & $1.0 \mathrm{a}$ & $1.5 \mathrm{a}$ & 4.5 & & $5.0 \mathrm{a}$ & $5.0 \mathrm{a}$ \\
\hline BAS 65100F 525SC + Dash; 7d & $1.2+0.50$ & $0.0 \mathrm{~b}$ & $0.0 \mathrm{~b}$ & 0.5 & $\mathrm{c}$ & $1.5 \mathrm{c}$ & 2.0 \\
\hline BAS 65100F 525SC; 7d & 1.00 & $0.0 \mathrm{~b}$ & $0.0 \mathrm{~b}$ & 0.5 & $\mathrm{c}$ & 1.5 & 2.5 \\
\hline BAS 65100F 525SC; 14d & 1.50 & $0.0 \mathrm{~b}$ & $0.8 \mathrm{ab}$ & 1.0 & $\mathrm{c}$ & $2.5 \mathrm{~b}$ & $5.0 \mathrm{a}$ \\
\hline BAS 65200F 56XL; 7d & 2.00 & 0.0 & $0.0 \mathrm{~b}$ & 0.5 & $\mathrm{c}$ & $1.5 \mathrm{c}$ & $3.5 \mathrm{bc}$ \\
\hline BAS 65200F 56XL; 7d & 2.50 & 0.0 & $0.0 \mathrm{~b}$ & 1.0 & c & 1.5 & $4.0 \mathrm{ab}$ \\
\hline BAS 65200F 56XL; 7d & 3.00 & 0.0 & $0.0 \mathrm{~b}$ & 1.0 & $\mathrm{c}$ & $2.0 \mathrm{bc}$ & $4.0 \mathrm{ab}$ \\
\hline Poliram 70WG; 7d & 2.00 & 0.0 & $0.0 \mathrm{~b}$ & 0.5 & $\mathrm{c}$ & $2.5 \mathrm{~b}$ & $4.0 \mathrm{ab}$ \\
\hline Acrobat MZ 90/600; 7d & 2.00 & 0.0 & $0.0 \mathrm{~b}$ & 0.5 & $\mathrm{c}$ & $1.5 \mathrm{c}$ & $2.5 \mathrm{~cd}$ \\
\hline
\end{tabular}

Los valores de rendimiento de papa comercial reflejan la eficiencia de los fungicidas en el control de los síntomas foliares. En las campañas 2008/09 (tabla 5) 
y 2009/10 (tabla 6) todos los tratamientos químicos superaron al testigo sin pulverizar, pero los mayores rendimientos en tubérculos comerciales se obtuvieron con BAS 65100F 525SC pulverizado cada 7 días; mientras que en rendimiento de tubérculos totales se observa la misma tendencia que en rendimiento de tubérculos comerciales; aunque BAS 65100F 525SC pulverizado cada 14 días y BAS 65200F 56XL en la dosis más alta cada 7 días no lograron superar el rendimiento del testigo sin tratar para esta variable.

Tabla 5. Rendimiento de papa recibo, "semilla" y total - 2008/09.

\section{R E N D I M I E N TO}

\begin{tabular}{lllllr} 
Tratamientos/dosis/intervalo & recibo & "semilla" & total & recibo & total \\
\hline Testigo sin tratar & $14.3 \mathrm{c}$ & $7.1 \mathrm{a}$ & $21.4 \mathrm{c}$ & 501 & 749 \\
BAS 65100F 525SC 0.8-7d. & $24.9 \mathrm{ab}$ & $4.2 \mathrm{~b}$ & $29.1 \mathrm{ab}$ & 872 & 1019 \\
BAS 65100F 525SC 1.0-7d. & $27.6 \mathrm{a}$ & $5.9 \mathrm{ab}$ & $33.5 \mathrm{a}$ & 966 & 1173 \\
Acrobat MZ 90/600 2.0-7d. & $22.5 \mathrm{~b}$ & $4.9 \mathrm{~b}$ & $27.4 \mathrm{~b}$ & 788 & 959 \\
BAS 65100F 525SC 1.0-14d. & $20.7 \mathrm{~b}$ & $5.1 \mathrm{~b}$ & $25.8 \mathrm{bc}$ & 725 & 903 \\
BAS 65100F 525SC 1.2-14d. & $23.9 \mathrm{ab}$ & $5.3 \mathrm{~b}$ & $29.2 \mathrm{ab}$ & 837 & 1022 \\
BAS 65200F 56XL 1.5-7d. & $22.4 \mathrm{~b}$ & $4.5 \mathrm{~b}$ & $26.9 \mathrm{~b}$ & 784 & 942 \\
BAS 65200F 56XL 2.0-7d. & $20.5 \mathrm{~b}$ & $5.0 \mathrm{~b}$ & $25.5 \mathrm{bc}$ & 718 & 893 \\
Poliram 80WG 2.5-7d. & $21.1 \mathrm{~b}$ & $5.8 \mathrm{ab}$ & $26.9 \mathrm{~b}$ & 739 & 942 \\
\hline
\end{tabular}

Los promedios que tienen letras en común no difieren entre sí $(P=0.05)$.

Tabla 6. Rendimiento de papa recibo, "semilla" y total - 2009/10.

\begin{tabular}{llllrr}
\hline & & \multicolumn{2}{c}{ R E N D I M I E N T O } \\
Tratamientos/intervalo/dosis & recibo & “semilla" & total & recibo & total \\
\hline Testigo sin tratar & $15.8 \mathrm{c}$ & $6.7 \mathrm{ab}$ & $22.5 \mathrm{c}$ & 617 & 878 \\
BAS 65100F 525SC + Dash 7d 0.8 & $15.0 \mathrm{c}$ & $7.0 \mathrm{a}$ & $22.8 \mathrm{c}$ & 585 & 890 \\
BAS 65100F 525SC 7d 1.0 & $28.5 \mathrm{a}$ & $6.1 \mathrm{ab}$ & $34.6 \mathrm{a}$ & 1113 & 1351 \\
Acrobat MZ 90/600 14d 2.0 & $23.8 \mathrm{~b}$ & $5.1 \mathrm{abc}$ & $28.9 \mathrm{~b}$ & 929 & 1129 \\
BAS 65100F 525SC 14d 1.0 & $22.0 \mathrm{~b}$ & $3.5 \mathrm{c}$ & $25.5 \mathrm{bc}$ & 859 & 996 \\
BAS 65100F 525SC 14d 1.2 & $22.3 \mathrm{~b}$ & $5.9 \mathrm{ab}$ & $28.2 \mathrm{~b}$ & 871 & 1101 \\
BAS 65200F 56XL 7d 1.5 & $23.1 \mathrm{~b}$ & $5.6 \mathrm{abc}$ & $28.7 \mathrm{~b}$ & 902 & 1121 \\
BAS 65200F 56XL 7d 2.0 & $21.0 \mathrm{~b}$ & $5.7 \mathrm{abc}$ & $26.7 \mathrm{bc}$ & 820 & 1043 \\
Poliram 80WG 7d 2.0 & $21.4 \mathrm{~b}$ & $4.5 \mathrm{bc}$ & $25.9 \mathrm{bc}$ & 836 & 1012 \\
& & & & & \\
\hline
\end{tabular}

Los promedios que tienen letras en común no difieren entre sí $(\mathrm{P}=0.05)$.

Durante el último año de evaluaciones, 2010/11 (tabla 7), los mayores rendimientos de tubérculos comerciales se obtuvieron con BAS 65100F 525SC + Dash, BAS 65100F 525SC, Acrobat MZ y BAS 65200F 56XL en la menor dosis, todos pulverizados cada 7 días, los cuales superaron a BAS 65100F 525SC pulverizado curativamente, BAS $65100 \mathrm{~F}$ 525SC cada 14 días y el testigo sin tratar. Una tendencia similar se observa al analizar el rendimiento de tubérculos totales. 
Tabla 7. Rendimiento de papa recibo, "semilla" y total - 2010/11.

\begin{tabular}{llllllr}
\hline Tratamientos/ dosis/ intervalo & Recibo & \multicolumn{1}{c}{ "semilla" } & total & \multicolumn{2}{c}{ recibo } & total \\
\hline Testigo sin tratar & $12.3 \mathrm{c}$ & $7.5 \mathrm{ab}$ & 19.8 & $\mathrm{~d}$ & 480 & 772 \\
BAS 65100F 525SC + Dash 1.2+0.50; 7d & $23.8 \mathrm{a}$ & 5.7 & $\mathrm{c}$ & $29.5 \mathrm{a}$ & 928 & 1150 \\
BAS 65100F 525SC 1.0; 7d & $21.9 \mathrm{ab}$ & 6.9 & $\mathrm{bc}$ & $28.8 \mathrm{ab}$ & 854 & 1084 \\
BAS 65100F 525SC 1.5;14d & $12.9 \mathrm{c}$ & $8.6 \mathrm{a}$ & 21.5 & $\mathrm{~cd}$ & 503 & 839 \\
BAS 65200F 56XL 2.0; 7d & $19.5 \mathrm{~b}$ & $6.6 \mathrm{bc}$ & $26.1 \mathrm{ab}$ & 761 & 975 \\
BAS 65200F 56XL 2.5; 7d & $18.0 \mathrm{~b}$ & 6.0 & $\mathrm{c}$ & $24.0 \mathrm{bc}$ & 702 & 936 \\
BAS 65200F 56XL3.0; 7d & $18.5 \mathrm{~b}$ & 5.9 & $\mathrm{c}$ & $24.4 \mathrm{bc}$ & 722 & 952 \\
Poliram 70WG 2.5; 7d & $18.4 \mathrm{~b}$ & 6.0 & $\mathrm{c}$ & $24.4 \mathrm{bc}$ & 718 & 952 \\
Acrobat MZ 90/600 2.5; 7d & $20.2 \mathrm{ab}$ & 7.0 & $\mathrm{bc}$ & $27.2 \mathrm{ab}$ & 788 & 1061 \\
\hline
\end{tabular}

* Los promedios que tienen letras en común no difieren entre sí $(\mathrm{P}=0.05)$.

Durante los tres años de evaluaciones, no se detectaron diferencias significativas entre dosis de aplicación de BAS 65200F $56 \mathrm{XL}$, tanto en eficacia de control de síntomas foliares como en rendimiento de tubérculos comerciales y totales.

Cuando analizamos el rendimiento de tubérculos "semilla" se observaron diferencias significativas entre tratamientos durante todo el período de evaluación. El testigo sin tratar registró siempre valores altos para esta variable y superó significativamente a algunos tratamientos químicos. Este resultado es lógico, ya que uno de los efectos más importantes que la enfermedad produce en el cultivo es la disminución del tamaño de los tubérculos.
Cuanto mayor es la eficacia de un fungicida para controlar el tizón tardío provoca un mayor rendimiento de tubérculos grandes (comerciales) en detrimento del rendimiento de tubérculos pequeños ("semilla").

En todo el período de evaluación los valores de aumento de rendimiento hallados en tubérculos totales son inferiores a los de tubérculos comerciales (tabla 8). Considerando éstos últimos, los valores observados con ametoctradin+dimetomorph llegan a $93 \%$, $80 \%$ y $93.5 \%$, en $2008 / 09,2009 / 10$ y 2010/11, mientras que en tubérculos totales llegan a 56.5\%, 53.7\% y $62.6 \%$, respectivamente. 
Tabla 8. Aumento de rendimiento de papa recibo, "semilla" y total - 2008/2009, 2009/2010 y 2010/11.

\begin{tabular}{lccc}
\hline Tratamientos/intervalo/dosis & recibo & "semilla" & total \\
\hline 2008/09 & & & \\
Testigo sin tratar & 0.0 & 0.0 & 0.0 \\
BAS 65100F 525SC 7d 0.8 & 74.1 & -40.8 & 36.0 \\
BAS 65100F 525SC 7d 1.0 & 93.0 & -17.0 & 56.5 \\
Acrobat MZ 90/600 14d 2.0 & 57.3 & -31.0 & 28.0 \\
BAS 65100F 525SC 14d 1.0 & 44.8 & -28.2 & 20.6 \\
BAS 65100F 525SC 14d 1.2 & 67.1 & -25.4 & 36.4 \\
BAS 65200F 56XL 7d 1.5 & 56.6 & -36.6 & 25.7 \\
BAS 65200F 56XL 7d 2.0 & 43.4 & -29.6 & 19.2 \\
Poliram 80WG 7d 2.0 & 47.6 & -18.3 & 25.7 \\
2009/10 & & & \\
Testigo sin tratar & 0.0 & 0.0 & 0.0 \\
BAS 65100F 525SC + Dash 7d 0.8 & -5.1 & 4.4 & 1.3 \\
BAS 65100F 525SC 7d 1.0 & 80.4 & -8.9 & 53.7 \\
Acrobat MZ 90/600 14d 2.0 & 50.6 & -23.9 & 28.4 \\
BAS 65100F 525SC 14d 1.0 & 39.2 & -47.8 & 13.3 \\
BAS 65100F 525SC 14d 1.2 & 41.1 & -12.0 & 25.3 \\
BAS 65200F 56XL 7d 1.5 & 46.2 & -16.5 & 27.5 \\
BAS 65200F 56XL 7d 2.0 & 32.9 & -15.0 & 18.6 \\
Poliram 80WG 7d 2.0 & 35.4 & -32.9 & 15.1 \\
2010/11 & & & \\
Testigo sin tratar & 0.0 & 0.0 & 0.0 \\
BAS 65100F 525SC + Dash 1.2+0.50; 7 & 93.5 & -24.0 & 72.5 \\
BAS 65100F 525SC 1.0; 7 & 78.0 & -8.0 & 62.6 \\
BAS 65100F 525SC 1.5;14 & 4.9 & 14.6 & 25.7 \\
BAS 65100F 525SC (curativo - 7); 1.5 & 0.0 & -8.0 & -3.5 \\
BAS 65200F 56XL 2.0; 7 & 58.5 & -12.0 & 52.6 \\
BAS 65200F 56XL 2.5; 7 & 46.4 & -20.0 & 40.4 \\
BAS 65200F 56XL3.0; 7 & 50.4 & -21.4 & 42.7 \\
Poliram 70WG 2.5; 7 & 49.5 & -20.0 & 59.1 \\
Acrobat MZ 90/600 2.5; 7 & 64.2 & -6.7 & \\
\hline & & & \\
\hline
\end{tabular}

\section{Conflictos de intereses}

Los autores declaran no tener conflictos con la publicación este trabajo de investigación.

\section{Referencias citadas}

Abad G., Abad J., 1995. Historical evidence on the occurrence of late blight of potato, tomato and pearl melon in the Andes of South America. Pages 36-49 in:Phytophthora infestans $150 . \mathrm{L}, \mathrm{J}$. Dowley, E. Bannon, L.R. Cooke, T. Keane and E. O'Sullivan, eds. Boole Press Ltd., Ireland.

Andrivon D., 1995. Comparison of race structure and diversity in populations of Phytophthora infestans, 1966-1993. Pages 71-76 in: Phytophthora infestans 150. L.J. Dowley, E. Bannon, L.R. Cooke, T. Keane and E. O'Sullivan, eds. Boole Press Ltd., Ireland.

Cox A. E., Large E. C., 1960. Potato blight epidemics throughout the world. ARS/USDA Handbook No. 174. 
Washington, D. C. US Gov. Printing Office.

Carmona D., Huarte M., Arias G., López A., Vincini A.M., Alvarez Castillo, $\mathrm{H}$, Manetti P., Chávez E., Torres M., Eyherabide J., Mantecón, J., Cichón L. and y Fernández D., 2003: Integrated Pest Management in Argentina, in Integrated Pest Management in the Global Arena, edited by $\mathrm{K} M$ Maredia, Institute of International Agriculture and Department of Entomology, Michigan State University, USA, D Dakouo, INERA, Burkina Faso (West Africa) and D Mota- Sanchez, Department of Entomology, Michigan State University, USA 24:313-326.

French E.R., Forbes G. y Landeo J., 1994. Ola migratoria de variantes más agresivas de Phytophthora infestans amenazan papa. Fitopatología 29: 15-18.

Fry W.E., Gooddwin S.B., Matuszak J.M., Spielman J. and Milgroom M.J., 1992. Population genetics and intercontinental migrations of Phytophthora infestans. Ann.Rev. Phyto. 30: 107-129.

Fry W.E., Goodwin S.B., Dyer A.T., Matuszak J.M., Drenth A., Tooley P.W., Sujkowskii L.S., Oh Y.J., Cohen B.A., Spielman L.J., Deahl K.L., Inglis D.A. and Sandlan K.P.,1993. Historical and recent migrations of Phytophthora infestans: Chronology, pathways, and implications. Plant Dis. 77: 653-661.

Hooker W. J., 1980. Compendio de Enfermedades de la Papa. (Versión española por Teresa Ames de Icochea.) The American Phytopathological Society, St. Paul, Minnesota, USA.

Huarte M., Van Damme M. y Macagno L., 1993. Importancia y nivel de daño económico de las principales enfermedades fungosas de la papa en la Argentina en relación con otros factores limitantes. In: Seminario Taller PROCIPA. "Control Integrado de las Principales Enfermedades Fungosas de la Papa" Bella Vista, Uruguay; Octubre 4-6.
Mantecón J.D., 2006: Potato late blight control with systemic fungicide using a disease prediction model in Argentina conditions, 2004:. Am. Phytho. Soc. Fung. Nem. 61: 144.

Mantecón J.D., 2006a: Potato late blight control with systemic fungicide using a disease prediction model in Argentina conditions, 2005. Am. Phytho. Soc. Fung. Nem. 61: 145.

Mantecón J.D., 2007: Potato Yield Increases Due to Fungicide Treatment in Argentinian Early Blight (Alternaria solani) and Late Blight (Phytophthora infestans) Field Trials during the 19962005 Seasons. Online. Plant Management Network. Plant Health Progress, February 2007.

Mantecón J.D., 2007a: Potato early blight control with fungicides on preventive program and long intervals applications. American Phytho. Soc. Plant Dis. Mana. Report. (1): 160.

Mantecón J.D., 2009: Importance of potato late blight in argentina. yield increases registered due to fungicidal control during twenty years. Ciencia e Investigación Agraria. 36 (1): 115-121.

Mantecón J.D., 2009a: Control of Potato early blight control with triazole fungicide using preventive and curative spraying, or a forecasting system. Ciencia e Investigación. Agraria 36 (2): 291-296.

Mantecón J.D., 2009b: Effect of fungicides sprays intervals on potato late blight control in Argentina, 2006. American Phytho. Soc. Plant Dis. Man. Report. (3): 140.

Niederhauser J.S. 1991. Phytophthora infestans: The Mexican connection. Pages 25-45 in: Phytophthora. J.A. Lucas, R.C. Shattock, D.S. Shaw and L.R. Cooke, eds. Br. Mycol. Soc. By Cambridge University, Cambrige. 